\title{
The New Extended Flexible Weibull Distribution and Its Applications
}

\author{
Zubair Ahmad, Zawar Hussain \\ Department of Statistics, Quaid-i-Azam University, Islamabad, Pakistan \\ Email address: \\ z.ferry21@gmail.com (Z.Ahmad), zhlangah@yahoo.com (Z. Hussain)
}

\section{To cite this article:}

Zubair Ahmad, Zawar Hussain. The New Extended Flexible Weibull Distribution and Its Applications. International Journal of Data Science and Analysis. Vol. 3, No. 3, 2017, pp. 18-23. doi: 10.11648/j.ijdsa.20170303.11

Received: June 7, 2017; Accepted: July 11, 2017; Published: September 26, 2017

\begin{abstract}
The present article considers a new function to propose a new lifetime distribution. The new distribution is introduced by mixing up a linear system of the two logarithms of cumulative hazard functions. The proposed model is called new extended flexible Weibull distribution and is able to model lifetime with bathtub shaped failure rates and offers greater flexibility. Therefore, it can be quite valuable to use an alternative model to other existing lifetime distributions, where, modeling of real data sets with bathtub shaped failure rates are of interest. A brief description of the statistical properties along with estimation of the parameters through maximum likelihood procedure are discussed. The potentiality of the proposed model is showed by discussing two real data sets. For these data sets, the proposed model outclasses the Flexible Weibull Extension, Inverse Flexible Weibull Extension and Modified Weibull distributions.
\end{abstract}

Keywords: Bathtub Shaped Failure Rates, Order Statistics, Moment Generating Function, Maximum Likelihood Estimation

\section{Introduction}

In the practice of analyzing real phenomena of nature one frequently uses the Rayleigh, Exponential, or Weibull distributions. These models possess numerous desirable properties and nice interpretations of its parameters enabling them to be utilized frequently. Between these lifetime models, Weibull distribution is the most prominent distribution for modeling real phenomena of nature. The Weibull model was originally introduced by Weibull [19], a Swedish physicist, and utilized it to represent the distribution of breaking strength of materials. Weibull model also has the shape and scale parameters, offering characteristics of both the exponential and Rayleigh distributions. In recent past years, the Weibull model becoming very popular in modeling lifetime data, because in the presence of censoring which makes it much easier to handle, at least numerically. The cumulative distribution function (CDF) of the Weibull model is given by.

$$
G(z)=1-e^{-\beta z^{\alpha}}, \quad z, \alpha, \beta>0 .
$$

The Weibull model is very useful in modeling real phenomena exhibiting monotonic failure rates. But, the Weibull model is inappropriate to use in modeling data having non-monotonic failure rates. Among non-monotonic failure rate function, the bathtub shaped failure rate is very useful and has a number of applications in the literature. For example, in bio-analysis the human mortality rate and in reliability engineering the lifecycle of electronic components is observed to have a bathtub shaped failure rate function. Due to practical utility in bio and reliability disciplines, numerous generalizations of Weibull distribution have been proposed in the literature aiming to improve its characteristics and to model real world scenario with nonmonotonic failure rate functions. These generalizations, including a statistical model with bathtub failure rate studied by Xie and Lai [20], Sarhan and Zaindin [17], Beta-Weibull (BW) distribution of Famoye et al. [11], Kumaraswamy Weibull (KW) distribution proposed by Cordeiro et al. [10], Generalized modified Weibull (GMW) distribution proposed by Carrasco et al. [9], Exponentiated modified Weibull extension (EMWEx) distribution introduced by Sarhan and Apaloo [16], Flexible Weibull (FWEx) distribution of Bebbington et al. [8], Generalized Flexible Weibull Extension (GFWEx) distribution studied by Ahmad and Iqbal [1], other extensions of Weibull model proposed by Ahmad and Hussain (2017) are [2], [3], [4], [5] and [6], respectively.

For a concise review of these distributions one may call to Pham and Lai [15] and Murthy et al. [14]. These distributions 
have numerous applications including reliability analysis, clinical studies, applied statistics and life testing experiments etc. Gurvich et al. [12] proposed a new class of aging distributions defined by the CDF given by.

$$
G(z)=1-e^{-\beta F(z)}, \quad z, \beta>0 .
$$

Where $F(z)$ is monotonically increasing function of $z$. It is a very useful method to mix two survival functions and create a new function as:

$$
S(z)=\eta_{1} S_{1}(z)+\left(1-\eta_{2}\right) S_{2}(z),
$$

Where $0<\eta_{1}, \eta_{2}<1$, this method of suggesting new functions is known as mixture of distributions, or

$$
S(z)=\eta_{1} S_{1}(z)+\psi S_{2}(z), \quad \eta_{1}, \psi>0 .
$$

One may also generate a new function by collaborating two cumulative hazard functions as:

$$
H(z)=\beta H_{1}(z)+\theta H_{2}(z),
$$

In term of cumulative hazard function (CHF), the CDF can be written as

$$
G(z)=1-e^{-H(z)}, \quad \mathrm{z}>0,
$$

where $H(z)$ fulfils the conditions stated below

a $H(z)$ is nonnegative and increasing function of $\mathrm{z}$

b $\lim _{z \rightarrow 0} H(z) \rightarrow 0$ and $\lim _{z \rightarrow \infty} H(z) \rightarrow \infty$.

The probability density function (PDF) associating to (5) has the following expression.

$$
g(z)=h(z) e^{-H(z)}, \quad z>0 .
$$

The modified Weibull distributions presented by Xie and Lai [20], Sarhan and Zaindin [17] and Almalki and Yaun [7] belongs to the class defined in (5). Here in (5), the $H(z)$ is bounded. On the other hand, in this article, effort have been made to produce a new function annoying to relax the boundary conditions. Hence, in this article $\log H(z)$ is used rather than $H(z)$. Because, it might be more exciting to use $\log H(z)$ rather $H(z)$ in order to advance a very flexible model. Hence, one may write (4) as

$$
H(z)=H_{1}^{\beta}(z) \times H_{2}^{\theta}(z),
$$

The expression provided in (6) can be re-write as

$$
\log H(z)=\beta \log H_{1}(z)+\theta \log H_{2}(z) .
$$

A mixture of the two logarithm of cumulative hazard functions, such as $z^{\gamma}$ and $z^{2}$ are proposed to introduce a new very flexible lifetime model. So, the expression given in (7), can be written in the following form.

$$
H(z)=e^{\beta z^{\gamma}+\theta z^{2}}
$$

By substituting (8) in (5), one can easily get the CDF of the new extended flexible Weibull (NEx-FW) distribution. The suggested model is capable of modeling data with bathtub failure rate. The present article is designed as: Section 2, offers the definition and graphical display of the new model. Section 3 , contains the basic statiatical properties. Section 4,5 and 6 , derives the moment generating function, probability generating function and factorial moment generating function of the NExFW distribution. Section 7 and 8, contains the estimation of the parameters and density functions of the order statistics. Section 9, offers the analysis to real data sets. Finally, section 10, contains concluding remarks.

\section{New Extended Flexible Weibull Distribution}

The CDF of the NEx-FW distribution is given by

$$
G(z ; \gamma, \beta, \theta)=\mathrm{P}-e^{-e^{\left(\beta z^{\gamma}+\theta z^{2}\right)}}, z, \gamma, \beta, \theta>0 .
$$

The probability distribution function (PDF) corresponding to (9) is given by

$$
g(z ; \gamma, \beta, \theta)=\left(\gamma \beta z^{\gamma-1}+2 \theta z\right) e^{\left(\beta z^{\gamma}+\theta z^{2}\right)} e^{-e^{\left(\beta z^{\gamma}+\theta z^{2}\right)}} .
$$

The survival function (SF) of the NEx-FW distribution is

$$
S(z ; \gamma, \beta, \theta)=? e^{-e^{\left(\beta_{z} \gamma^{\gamma}+\theta z^{2}\right)}},
$$

with HF

$$
h(z ; \gamma, \beta, \theta)=\left(\gamma \beta z^{\gamma-1}+2 \theta z\right) e^{\left(\beta z^{\gamma}+\theta z^{2}\right)} .
$$

The figure 1 displays the HF of the NEx-FW distribution for different values of parameters.

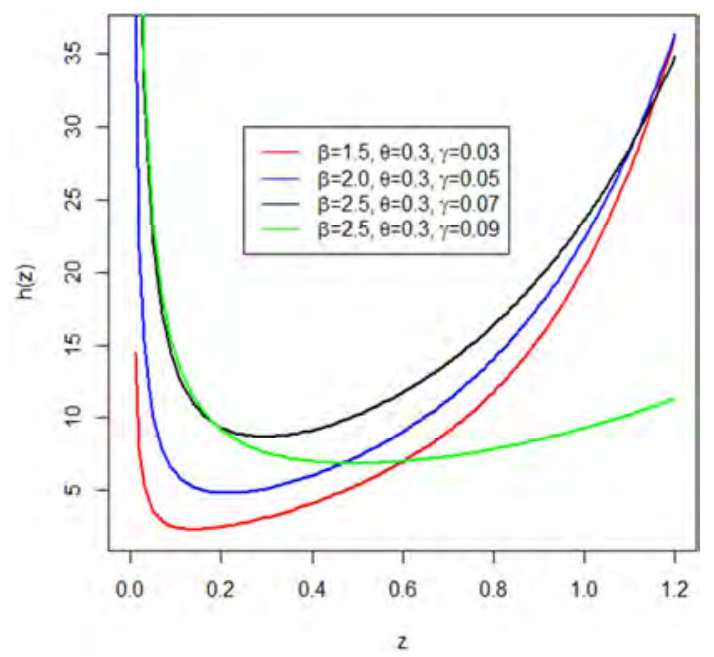

Figure 1. HF of the New Extended Flexible Weibull distribution, for different values of parameters. 


\section{Basic Properties}

This section of the paper covers the basic statistical properties of the NEx-FW distribution.

\subsection{Quantile and Median}

The expression for the $q^{\text {th }}$ quantile $z_{q}$ of the NEx-FW distribution is given by

$$
\beta z_{q}^{\gamma}+\theta z_{q}^{2}-\log \{-\log (1-q)\}=0 .
$$

Using $q=0.50$, in (11), one can easily obtain the median of the NEx-FW distribution. Also, putting $q=0.25$, and $q=0.75$, in (11), one may get the $1^{s t}$ and $3^{\text {rd }}$ quartiles of the NEx-FW distribution, respectively.

\subsection{Generation of Random Numbers}

The formula for generating random numbers from NExFW distribution can be derived as

$$
G(z ; \gamma, \beta, \theta)=u
$$

where $u \sim U(0,1)$. After simplification, this yields

$$
\begin{aligned}
& \beta z^{\gamma}+\theta z^{2}-\log \{-\log (1-u)\}=0 . \\
& \mu_{r}^{\prime}=\sum_{i=0}^{\infty} \sum_{j=0}^{\infty} \frac{(-1)^{i}(i+1)^{i} \beta^{j}}{i ! j !}\left\{\gamma \beta \int_{0}^{\infty} z^{r+\gamma(j+1)-1} e^{\theta(i+1) z^{2}} d z+2 \theta \int_{0}^{\infty} z^{r+j \gamma-1} e^{\theta(i+1) z^{2}} d z\right\} .
\end{aligned}
$$

Using the definition of gamma function (Zwillinger [21]) in the following form,

$$
\Gamma z=x^{z} \int_{0}^{\infty} t^{z-1} e^{t x} d t, \quad z, x>0 .
$$

Using the above definition of gamma function in (12), and finally, one may get

$$
\mu_{r}^{\prime}=\sum_{i=0}^{\infty} \sum_{j=0}^{\infty} \frac{(-1)^{i}(i+1)^{i} \beta^{j}}{i ! j !}\left\{\gamma \beta \frac{\Gamma\left(\frac{r+\gamma(j+1)}{2}\right)}{2(\theta(i+1))^{\frac{r+j(\gamma+1)}{2}}}+\theta \frac{\Gamma\left(\frac{r+j \gamma+2}{2}\right)}{(\theta(i+1))^{\frac{r+j \gamma+2}{2}}}\right\}
$$

\section{Moment Generating Function}

If $\mathrm{Z} \sim \mathrm{NEx}-\mathrm{FW}(z ; \gamma, \beta, \theta)$, then the moment generating function of $Z$ is derived as

$$
\begin{gathered}
M_{z}(t)=\int_{0}^{\infty} e^{t z} g(z ; \gamma, \beta, \theta) d z \\
M_{z}(t)=\sum_{r=0}^{\infty} \frac{t^{r}}{r !} \int_{0}^{\infty} z^{r} g(z ; \gamma, \beta, \theta) d z
\end{gathered}
$$

$$
M_{z}(t)=\sum_{r=0}^{\infty} \frac{t^{r}}{r !} \mu_{r}^{\prime} .
$$

By using (13), in (14), one may have the proof of the NExFW distribution.

\section{Probability Generating Function}

The probability generating function (PGF) of NEx-FW distribution is derived

$$
G(\gamma)=\int_{0}^{\infty} \gamma^{z} g(z ; \gamma, \beta, \theta) d z
$$




$$
\begin{aligned}
G(\gamma) & =\sum_{r=0}^{\infty} \frac{\log ^{r}(\gamma)}{r !} \int_{0}^{\infty} z^{r} g(z ; \gamma, \beta, \theta) d z \\
G(\gamma) & =\sum_{r=0}^{\infty} \frac{\log ^{r}(\gamma)}{r !} \mu_{r}^{\prime} .
\end{aligned}
$$

On substituting (13), in (15), one may get the expression for the PGF of NEx-FW distribution.

\section{Factorial Moment Generating Function}

The factorial moment generating function (FMGF) of NEx-FW distribution can be derived as

$$
\begin{aligned}
& H_{0}(1+\delta)=\int_{0}^{\infty}(1+\delta)^{z} g(z ; \gamma, \beta, \theta) d z \\
& \ln L=\sum_{i=1}^{k} \log \left(\gamma \beta z_{i}^{\gamma-1}+2 \theta z_{i}\right)+\sum_{i=1}^{k}\left(\beta z_{i}^{\gamma} \mathfrak{Y} \boldsymbol{\theta} z_{i}^{2}\right)-\sum_{i=1}^{k} e^{\left(\beta z_{i}^{\gamma}+\theta z_{i}^{2}\right)} .
\end{aligned}
$$

By attaining the partial derivatives of the expression in (17) on parameter, and then equating to zero, one may have

$$
\begin{gathered}
\frac{d \ln L}{d \beta}=\sum_{i=1}^{k} \frac{\gamma z_{i}^{\gamma-1}}{\left(\gamma \beta z_{i}^{\gamma-1}+2 \theta z_{i}\right)}+\sum_{i=1}^{k} z_{i}^{\gamma}-\sum_{i=1}^{k} z_{i}^{\gamma} e^{\left(\beta z_{i}^{\gamma}+\theta z_{i}^{2}\right)} . \\
\frac{d \ln L}{d \theta}=\sum_{i=1}^{k} \frac{2 z_{i}}{\left(\gamma \beta z_{i}^{\gamma-1}+2 \theta z_{i}\right)}+\sum_{i=1}^{k} z_{i}^{2}-\sum_{i=1}^{k} z_{i}^{2} e^{\left(\beta z_{i}^{\gamma}+\theta z_{i}^{2}\right)} . \\
\frac{d \ln L}{d \gamma}=\beta \sum_{i=1}^{k} \frac{\left(\gamma z_{i}^{\gamma-1} \log \left(z_{i}\right)+z_{i}^{\gamma-1}\right)}{\left(\gamma \beta z_{i}^{\gamma-1}+2 \theta z_{i}\right)}+\beta \sum_{i=1}^{k} z_{i}^{\gamma} \log \left(z_{i}\right)-\beta \sum_{i=1}^{k} e^{\left(\beta z_{i}^{\gamma}+\theta z_{i}^{2}\right)} z_{i}^{\gamma} \log \left(z_{i}\right) .
\end{gathered}
$$

It is perceived that, the expressions given in (18)-(20) do not hold solution in closed forms; so, the estimates of the unknown parameters can be obtained numerically by using the iterating procedure. The "SANN" algorithm in R language is used to estimate the parameters numerically.

\section{Order Statistic}

Consider a sample say $Z_{1}, Z_{2}, \cdots, Z_{k}$ selected randomly from NEx-FW distribution with parameters $(\gamma, \beta, \theta)$, having ordered values $Z_{1: k}, Z_{2: k}, \cdots, Z_{k: k}$. Let $Z_{(1: k)}$ represents the smallest of $Z_{1: k}, Z_{2: k}, \cdots, Z_{k: k}$, similarly, $Z_{(2: k)}$ represents the second smallest of $Z_{1: k}, Z_{2: k}, \cdots, Z_{k: k}$ and $Z_{(k: k)}$ represents the $k^{\text {th }}$ smallest of $\left\{Z_{1: k}, Z_{2: k}, \cdots, Z_{k: k}\right\}$. Then, the PDF of $Z_{(i: k)}$, $1 \leq i \leq k$ is given by

$$
g_{i: k}(z)=\frac{1}{\operatorname{beta}(i, k-i+1)} g(z)[G(z)]^{i-1}[1-G(z)]^{k-i} .
$$

So, the PDF of smallest order statistic is

$$
g_{1: k}(z)=k\left(\gamma \beta z_{1}^{\gamma-1}+2 \theta z_{1}\right) e^{\left(\beta z_{1}^{\gamma}+\theta z_{1}^{2}\right)}\left(e^{-e^{\left(\beta z_{1}^{\gamma}+\theta z_{1}^{2}\right)}}\right)^{k} .
$$

Also, the PDF of largest order statistic is

$$
g_{k: k}(z)=k\left(\gamma \beta z_{k}^{\gamma-1}+2 \theta z_{k}\right) e^{\left(\beta z_{k}^{\gamma}+\theta z_{k}^{2}\right)} e^{-e^{\left(\beta z_{k}^{\gamma}+\theta z_{k}^{2}\right)}}\left(1-e^{-e^{\left(\beta z_{k}^{\gamma}+\theta z_{k}^{2}\right)}}\right)^{k-1} .
$$

\section{Applications}

In this section, two real life application are presented. The result of the goodness of fit of the suggested model is compared with three other well-known competing lifetime models. The investigative tools such as Akaike's Information 
Criterion (AIC), Hannan-Quinn information criterion (HQIC), Cramer-von-Misses (CM) test statistics, Anderson-Darling (AD) test statistic, Consistent Akaike's Information Criterion (CAIC), Bayesian information criterion (BIC), KolmogorovSmirnov (K-S) test statistic and $\log$ likelihood $-2 l(., z)$ are considered. On the basis these measures, it is showed that the new model provides greater flexibility.

\subsection{Example 1}

The first data set obtained from Tahir et al. [18], denotes the failure times of 84 Aircraft Windshield. The failure times are as: $0.040,1.866,2.385,3.443,0.301,1.876,2.481,3.467$,
$0.309,1.899,2.610,3.478,0.557,1.911,2.625,3.578,0.943$, $1.912,2.632,3.595,1.070,1.914,2.646,3.699,1.124,1.981$, $2.661,3.779,1.248,2.010,2.688,3.924,1.281,2.038,2.82,3$, $4.035,1.281,2.085,2.890,4.121,1.303,2.089,2.902,4.167$, $1.432,2.097,2.934,4.240,1.480,2.135,2.962,4.255,1.505$, 2.154, 2.964, 4.278, 1.506, 2.190, 3.000, 4.305, 1.568, 2.194, $3.103,4.376,1.615,2.223,3.114,4.449,1.619,2.224,3.117$, $4.485,1.652,2.229,3.166,4.570,1.652,2.300,3.344,4.602$, $1.757,2.324,3.376$ and 4.663 . The final results, after applying the proposed distribution along with the competing models are provided in example 1 , are summarized in table $1 \& 2$.

Table 1. Goodness of fit results for NEx-FW, FWEx, IFWEx and MW.

\begin{tabular}{llllll}
\hline Dist. & Max. Likelihood Estimates & AD & CM & KS & $-2 \log l$ \\
\hline NEx-FW & $\hat{\gamma}=0.115, \hat{\beta}=1.400, \hat{\theta}=1.959$ & 1.976 & 0.339 & 0.117 & 134.682 \\
FWEx & $\hat{\beta}=0.307, \hat{\theta}=1.396$ & 5.575 & 0.897 & 0.320 & 175.828 \\
IFWEx & $\hat{\beta}=0.0643, \hat{\theta}=0.498$ & 1.820 & 0.225 & 0.4857 & 187.242 \\
MW & $\hat{\beta}=2.798, \hat{\sigma}=0.044, \hat{\alpha}=1.0260$ & 0.5267 & 0.0567 & 0.666 & 275.07 \\
\hline
\end{tabular}

Table 2. Goodness of fit results for NEx-FW, FWEx, IFWEx and MW.

\begin{tabular}{lllll}
\hline Dist. & AIC & BIC & CAIC & HQIC \\
\hline NEx-FW & 275.36 & 282.69 & 275.66 & 278.312 \\
FWEx & 355.655 & 360.5412 & 355.802 & 357.620 \\
IFWEx & 378.48 & 383.371 & 378.632 & 380.450 \\
MW & 556.155 & 563.483 & 556.451 & 559.1026 \\
\hline
\end{tabular}

\subsection{Example 2}

The second data set denotes the failure times of a sample of 30 devices taken from Khan and Jan [13]. The times are 2.75, $0.13,1.47,0.23,1.81,0.30,0.65,0.10,3.00,1.73,1.06,3.00,3.00,2.12,3.00,3.00,3.00,0.02,2.61,2.93,0.88,2.47,0.28$, $1.43,3.00,0.23,3.00,0.80,2.45$ and 2.66 . The final goodness of fit result of the proposed model along with the competing models, are summarized in table $3 \& 4$.

Table 3. Goodness of fit results for NEx-FW, FWEx, IFWEx and MW.

\begin{tabular}{llllll}
\hline Dist. & Max. Likelihood Estimates & AD & CM & KS & -2 logl \\
\hline NEx-FW & $\hat{\gamma}=0.301, \hat{\beta}=1.400, \hat{\theta}=0.196$ & 1.02 & 0.14 & 0.163 & 35.11 \\
FWEx & $\hat{\beta}=0.3283, \hat{\theta}=0.1610$ & 2.060 & 0.326 & 0.3937 & 53.6561 \\
IFWEx & $\hat{\beta}=0.029, \hat{\theta}=0.621$ & 1.600 & 0.242 & 0.2907 & 187.242 \\
MW & $\hat{\beta}=4.797, \hat{\theta}=0.0082, \hat{\alpha}=1.24672$ & 1.3077 & 0.200 & 0.4727 & 75.951 \\
\hline
\end{tabular}

Table 4. Goodness of fit results for NEx-FW, FWEx, IFWEx and MW.

\begin{tabular}{lllll}
\hline Dist. & AIC & BIC & CAIC & HQIC \\
\hline NEx-FW & 76.22 & 80.42 & 77.14 & 77.56 \\
FWEx & 111.3122 & 114.114 & 111.756 & 112.208 \\
IFWEx & 106.341 & 109.144 & 106.786 & 107.2381 \\
MW & 157.90 & 162.106 & 158.826 & 159.247 \\
\hline
\end{tabular}

\section{Conclusion}

In this paper, a new lifetime distribution entitled New Extended Flexible Weibull Distribution is introduced by taking into account a linear system of the two logarithms of cumulative hazard functions. The suggested model offers greater distribution flexibility and is able to model lifetime data with bathtub shaped failure rates. A concise explanation of the mathematical properties of the proposed model, with estimation of parameters using maximum likelihood procedure are discussed. The proposed modal is illustrated by means of analyzing two real data sets, and the goodness fit of the proposed model is compared with that of three other existing lifetime distributions. Analyzing these two data sets, it is observed that the new model provides best fit than the 
competitive models. It is hoped that the New Extended Flexible Weibull distribution will serve as one of the most useful lifetime model and will attract a wide range of practical applications in the field of bio-medical and reliability engineering.

\section{Acknowledgements}

The authors are so grateful to the editor and anonymous referees for a careful checking of the details and for the helpful comments on an earlier version of the paper that improved the presentation in the paper. On behalf of corresponding author of this article, I would like to thank my respected parents for allowing me to realize my own potential. Also, I need to thank my sweet sister, who taught me the value of handwork \& education. Without her, I may never have gotten to where I am today.

\section{References}

[1] Ahmad, Z. and Iqbal, B. (2017). Generalized Flexible Weibull Extension Distribution. Circulation in Computer Science, Volume 2(4), 68-75. https:/doi.org/10.22632/css-2017-252-11.

[2] Ahmad, Z. and Hussain, Z. (2017). New Flexible Weibull Distribution. International Journal of Advanced Research in Electrical, Electronics and Instrumentation Engineering, Vol.6, No.13.

[3] Ahmad, Z. and Hussain, Z. (2017). Modified New Flexible Weibull Distribution. Circulation in Computer Science, Vol.2, No.6, pp: (7-13). https://doi.org/10.22632/ccs-2017-252-30.

[4] Ahmad, Z. and Hussain, Z. (2017). New Extended Weibull Distribution. Circulation in Computer Science, Vol.2, No.6, pp: (14-19). https://doi.org/10.22632/ccs-2017-252-31.

[5] Ahmad, Z. and Hussain, Z. (2017). Flexible Weibull Distribution. Journal of Computer and Mathematical Science, Vol.8, No.6, pp: (251-260).

[6] Ahmad, Z. and Hussain, Z. (2017). Very Flexible Weibull Distribution. Mayfeb Journal of Mathematics. (Accepted).

[7] Almalki, S. J. and Yuan, J. (2013). A new modified Weibull distribution. Reliability Engineering and System Safety, 111, 164-170.

[8] Bebbington, M., Lai, C. D. and Zitikis, R. (2007). A flexible Weibull extension. Reliability Engineering and System Safety, 92, 719-726.

[9] Carrasco M., Ortega E. M. and Cordeiro G. M. (2008). A generalized modified Weibull distribution for lifetime modeling. Computational Statistics and Data Analysis, 53(2), 450-62.

[10] Cordeiro, G. M., Ortega, E. M. and Nadarajah, S. (2010). The
Kumaraswamy Weibull distribution with application to failure data. Journal of the Franklin Institute, 347, 1399-429.

[11] Famoye, F., Lee, C. and Olumolade, O. (2005). The betaWeibull distribution. Journal of Statistical Theory and Applications, 4(2), 121-36.

[12] Gurvich, M. R., Dibenedetto, A. T. and Rande, S. V. (1997). A new statistical distribution for characterizing the random length of brittle materials. J Mater Sci; 32:2559-64.

[13] Khan, A. H. and Jan, T. R. (2016). The new modified generalized linear failure rate distribution. J. Stat. Appl. Pro. Lett. 3, No. 2, 83-95.

[14] Murthy, D. N. P., Xie, M. and Jiang, R. (2003). Weibull Models. John Wiley and Sons, New York.

[15] Pham, H. and Lai, C. D. (2007). On recent generalizations of the Weibull distribution. IEEE Transactions on Reliability, 56, 454-8.

[16] Sarhan, A. M. and Apaloo, J. (2013). Exponentiated modified Weibull extension distribution. Reliability Engineering and System Safety, 112, 137-144.

[17] Sarhan, A. M. and Zaindin, M. (2009). Modified Weibull distribution. Applied Sciences, 11, 123-136.

[18] Tahir, M. H., Cordeiro, G. M., Mansoor, M. and Zubair, M. (2015). The Weibull-Lomax Distribution: Properties and Applications', Hacettepe Journal of Mathematics and Statistics.

[19] Weibull, W. (1939). A statistical theory of the strength of material. Ingeniors Vetenskaps Akademiens, Stockholm 151.

[20] Xie, M. and Lai, C. D. (1996). Reliability analysis using an additive Weibull model with bathtub-shaped failure rate function. Reliability Engineering and System Safety, 52(1), 87-93.

[21] Zwillinger, D. (2014). Table of integrals, series, and products. Elsevier.

\section{Biography}

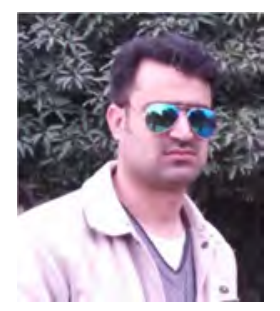

Zubair Ahmad S/O Wali Muhammad, research scholar at Quaid-i-Azam University, obtained his Master degree in Statistics in 2014 at University of Malakand and received his M. Phil. Degree in Statistics in 2017 at Quaid-i-Azam University, Islamabad, Pakistan. His research topic in M. Phil. was "On Different Modifications of Weibull Distribution" under the guidance \& supervision of Dr. Zawar Hussain, Quaid-i-Azam University, Islamabad, Pakistan. 